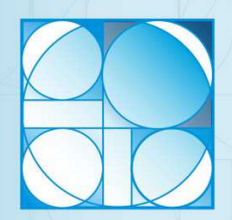

$2^{\circ}$ Prêmio Nacional de Ensino de Bioquímica e Biologia Molecular

Sociedade Brasileira de Bioquímica

e Biologia Molecular - SBBq
Journal of Biochemistry Education

Revista de Ensino de Bioquímica

Revista de Enseñanza de Bioquímica

Q4. Sociedade Brasileira de Bioguimica

ISSN: 2318-8790

ISSN (until 2012): 1677-2318

\title{
Vitamins and Coenzymes: An Interactive Study
}

\author{
Trindade, V.M.T; Menegasso, J.L. M.; Salbego, C.G.
}

Departamento de Bioquímica, Instituto de Ciências Básicas da Saúde, Universidade Federal do Rio Grande do Sul, Porto Alegre, RS, Brazil.

Introduction: Vitamins form a group of organic micronutrients without chemical relation, whose biosynthesis in humans is nonexistent or insufficient, being required in the diet. They have specific cellular functions and are precursors of coenzymes in the intermediate metabolism. They are classified as water-soluble and lipid-soluble. Water-soluble substances are subdivided into the group known as "non-complex B" and "B complex". The last one has three subgroups: vitamins that act on the energy metabolism (B1, B2, B3, B5 and B7), vitamins that act on hematopoietic metabolism (B9, and B12) and others (B6). Objectives: Considering the scarcity of interactive materials in the subject, this educational object aims to present interactively $B$ vitamins showing: their chemical structures with their respective coenzyme forms, the reactions in which they are involved, their action mechanisms, food sources and consequences of their deficiencies. Materials and Methods: This material was developed with PHP web language, associated with HTML, CSS and Javascript for the creation and insertion of figures, hypertexts and animations. A questionnaire about interactivity, functionality and esthetics characteristics and general information levels was used for its evaluation. Results and Discussion: This educational resource presents web page properties, including the possibility of access by mobile devices. It is composed by flaps referring to the visual identity, tutorial and credits, followed by a start button that reveals a menu subdivided into 10 flaps corresponding to a conceptual introductory text, vitamins B1, B2, B3, B5, B6, B7, B9, B12 and an interactive questionnaire (www.ufrgs.br/vitaminas-b). The functionality, design, language, and interactivity characteristics were rated as excellent by $80 \%$ of basic biochemistry students. Conclusion: This digital educational resource can be considered an adequate teaching-learning tool, easily accessible to students in the health area, as well as to the general public.

Keywords: vitamins, coenzymes, basic biochemistry.

Support: Secretaria de Educação à Distância; UFRGS. 